\title{
Improving MIMO-OFDM decision-directed channel estimation by utilizing error-correcting codes
}

\author{
P. Beinschob, M. Lieberei, and U. Zölzer \\ Department of Signal Processing and Communications, Helmut-Schmidt-Universität/University of the Federal Armed Forces \\ Hamburg, Germany
}

\begin{abstract}
In this paper a decision-directed Multiple-Input Multiple-Output (MIMO) channel tracking algorithm is enhanced to raise the channel estimate accuracy. While DDCE is prone to error propagation the enhancement employs channel decoding in the tracking process. Therefore, a quantized block of symbols is checked on consistency via the channel decoder, possibly corrected and then used. This yields a more robust tracking of the channel in terms of bit error rate and improves the channel estimate under certain conditions.

Equalization is performed to prove the feasibility of the obtained channel estimate. Therefore a combined signal consisting of data and pilot symbols is sent. Adaptive filters are applied to exploit correlations in time, frequency and spatial domain. By using good error-correcting coding schemes like Turbo Codes or Low Density Parity Check (LDPC) codes, adequate channel estimates can be acquired even at low signal to noise ratios (SNR). The proposed algorithm among two others is applied for channel estimation and equalization and results are compared.
\end{abstract}

\section{Introduction}

MIMO-OFDM combines two powerful techniques for next generation wireless communication systems. For development accurate channel estimates of certain environments are required to derive realistic parameters for Monte-Carlo simulations of MIMO channels. They can be obtained by applying a MIMO system and an appropriate channel estimation algorithm in the respective environment. In Akhtman and Hanzo (2007) a channel tracking algorithm based upon a MIMO Recursive Least Square (RLS) algorithm is proposed. MIMO RLS channel estimation has obvious advantages over

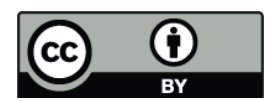

Correspondence to: $\mathrm{P}$. Beinschob (patric.beinschob@hsu-hamburg.de) traditional interpolation approaches. On the one hand, time and spatial diversity can be exploited which makes it suitable for spatial multiplexing MIMO system design approaches even in fast time-varying environments. On the other hand, due to the filter structure the output depends only on the current input, therefore the channel estimate is available almost delay-free rather then after whole frame processing as in interpolation if time variance is reflected.

The paper is structured as follows. The system model is described in Sect. 2. The employed MIMO-OFDM RLS algorithm is reviewed in Sect. 3. The enhancement to this algorithm is shown in Sect. 4. Finally, the performance of Turbo Codes and LDPC Codes as channel coding schemes in this context is compared using the mean square error of the channel estimate and the MIMO system's bit error rate (BER) versus SNR in Sect. 5, before concluding in Sect. 6.

\section{System model}

The vector of received values $\mathbf{r}$ at the time sample $m$ of a MIMO system is a superposition of $L \cdot N_{T}$ previously send samples and the current $N_{T}$ samples. It is given by

$\mathbf{r}[m]=\sum_{l=0}^{L} \mathbf{H}[l, m] \cdot \mathbf{s}[m-l]+\mathbf{w}[m]$,

where $\mathbf{s}[m]$ denotes the current vector of symbols of every transmit antenna, $\mathbf{w}$ an identically, independently distributed (i.i.d.) additive white Gaussian noise term and $\mathbf{H}$ the MIMO channel matrix. The past sent samples are denoted by $\mathbf{s}[m-l]$, for $l \neq 0$. For simulations the data symbols of the $K$ subcarrier are modulated by an inverse Fast Fourier Transform (IFFT). Every value corresponding to a transmit antenna of the resulting vectors is transmitted using the formula above. If a time variant channel is assumed, for every sample $m$ the channel matrix differs from the previous one. It is modeled such that the movement of the terminal results

Published by Copernicus Publications on behalf of the URSI Landesausschuss in der Bundesrepublik Deutschland e.V. 
in a Doppler shift of the send signal. This property is integrated in $\mathbf{H}[l, m]$ by first generating independent channel matrices from every $m, l$ and second filtering over time $m$ for every tap $l$ with the Jakes spectrum, that reflects the spread of the Doppler shifts in the frequency domain. In a simulation only a finite number of Doppler frequencies can be realized, therefore the Jakes spectrum is sampled at 16 non-zero frequencies. The result is a continuously time-varying channel, even for small terminal velocities (Pätzold, 1999).

In the frequency domain the system model in Eq. (1) can be described in the time invariant case as

$\mathbf{r}[n, k]=\mathbf{H}[n, k] \cdot \mathbf{s}[n, k]+\mathbf{w}[n, k]$,

where $n$ denotes the time index of an OFDM symbol and $k$ its subcarrier index. The MIMO channel coefficients $H_{r, t}, r=1, \ldots, n_{R}, t=1, \ldots, n_{T}$ are normal i.i.d. random variables for every tap. As described above they may vary over time and frequency $n, k$ in a MIMO-OFDM frame. Through the whole discussion and in the simulations a frame consists of 256 OFDM symbols of which the first 128 are pilots to train the adaptive filters. The source bits are coded using modern coding schemes like Turbo Codes and LDPC Codes. The codes are constructed such that one codeword fits in an OFDM symbol. Thus for a $M$-QAM modulation scheme and code rate $R_{C}$ the codeword length set to

$n_{C}=K \cdot R_{C} \cdot \log _{2} M$

It is important for the ability to track time-variant channels that the symbols in a transmitted OFDM symbol can be decoded at once.

For all simulations presented pilots and data symbols are send to compute a bit error rate as a confidence level for the estimated channel impulse response.

The pilot tones are organized as in code division multiplexing (CDM) manner. A single symbol is mapped to a sequence. The sequences are pairwise orthogonal thus preventing mutual interference. Each transmit antenna sends its own sequence. A set of orthogonal sequences are (Frank-ZadoffChu, 1972) sequences which are used for channel estimation in the training phase.

\section{Adaptive MIMO RLS channel estimation}

The employed MIMO-OFDM-Recursive Least Squares (RLS) algorithm, proposed in Akhtman and Hanzo (2007), is due to find a solution to the Wiener-Filter problem:

$\min E\left\{(\mathbf{r}[k]-\mathbf{H}[k] \cdot \mathbf{s}[k])^{2}\right\}$.

The global solution is called Wiener-Hopf equation

$\mathbf{H}[k]=\left(\boldsymbol{\Phi}^{-1}[k] \boldsymbol{\theta}[k]\right)^{H}$. with

$$
\begin{array}{ll}
\boldsymbol{\Phi}[k]=E\left\{\mathbf{s}[k] \cdot \mathbf{s}^{H}[k]\right\} & \forall k \\
\boldsymbol{\theta}[k]=E\left\{\mathbf{s}[k] \cdot \mathbf{r}^{H}[k]\right\} & \forall k .
\end{array}
$$

In general the expectation values are unknown and must be estimated. To derive the RLS algorithm Eq. (4) is modified to get the following optimization problem: The channel estimate $\tilde{\mathbf{H}}[n, k]$ at time instant $n$ on subcarrier $k$ should map the data symbols $\mathbf{s}[n, k]$ in such a way that the distance to the actually received symbols is minimized

$\min \varepsilon=\min \|\tilde{\mathbf{r}}[n, k]-\tilde{\mathbf{H}}[n, k] \cdot \mathbf{s}[n, k]\|_{2}$.

The RLS implementation of this solution replaces the expectation value with an exponentially weighted sum. Therefore the matrices can be calculated recursively:

$$
\begin{aligned}
\boldsymbol{\Phi}[n, k] & =\sum_{m=1}^{n} \xi^{n-m} \mathbf{s}[m, k] \mathbf{s}^{H}[m, k] \\
& =\xi \boldsymbol{\Phi}[n-1, k]+\mathbf{s}[n, k] \mathbf{s}^{H}[n, k]
\end{aligned}
$$

and

$$
\begin{aligned}
\boldsymbol{\theta}[n, k] & =\sum_{m=1}^{n} \xi^{n-m} \mathbf{s}[m, k] \mathbf{r}^{H}[m, k] \\
& =\xi \boldsymbol{\theta}[n-1, k]+\mathbf{s}[n, k] \mathbf{r}^{H}[n, k] .
\end{aligned}
$$

Still Eq. (5) is the global minimum and used to calculate the channel matrix $\mathbf{H}$. By the $\xi$ weighting, newer samples have greater impact on the channel estimate thus adaptivity on time variant channels can be achieved. For lower SNR a $\xi \rightarrow 1$ is in favor of averaging noise out but the algorithm loses its ability to adapt to time varying channels (Haykin, 2001). So a compromise has to be found, it is set to $\xi:=0.95$.

By employing such an estimator for every subcarrier, the complete channel transfer function $\mathbf{H}_{r, t}^{k}[n]$ for every SISO channel of the MIMO system is calculated for every OFDM symbol.

Next step is to distinct the corresponding meaningful CIR taps from noise. This is done by an eigenvalue tracking algorithm called Projection Approximation Subspace Tracking (PAST) (Yang, 1995). The meaningful CIR taps build a subspace in the signal space which is reasonably smaller than the signal space. In tracking only the subspace complexity can be reduced especially in focus on the third step, in which the CIR taps are predicted for the next OFDM symbol.

The CIR subspace $\boldsymbol{\alpha}_{r, t}^{l}[n]$ can be calculated using an RLS algorithm which minimizes the following cost function

$$
\min _{\mathbf{W}} J(\mathbf{W})=\mathrm{E}\left\{\left\|\mathbf{H}-\mathbf{W} \mathbf{W}^{H} \mathbf{H}\right\|^{2}\right\},
$$

in which $\mathbf{W}$ denotes the subspace transformation matrix. Again replacing expectation value with an exponentially 


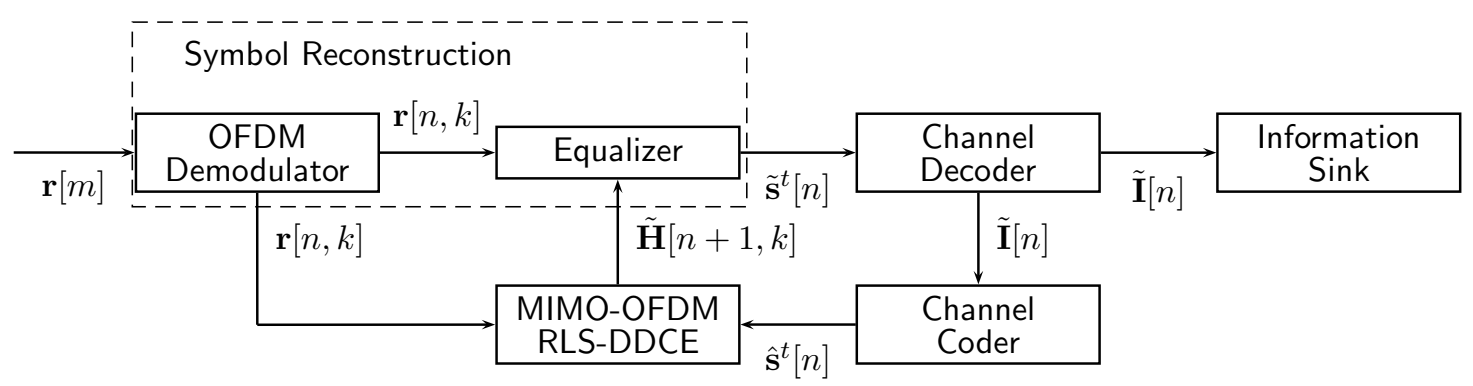

Fig. 1. Block diagram of proposed algorithm enhancement.

weighted sum yields

$$
\begin{aligned}
J(\mathbf{W}[n]) & =\mathrm{E}\left\{\left\|\mathbf{H}[n]-\mathbf{W}[n] \mathbf{W}^{H}[n] \mathbf{H}[n]\right\|^{2}\right\} \\
& =\sum_{i=1}^{n} \eta^{n-i}\left\|\mathbf{H}[i]-\mathbf{W}[i] \mathbf{W}^{H}[i] \mathbf{H}[m]\right\|^{2} .
\end{aligned}
$$

For $\boldsymbol{\alpha}[n]=\mathbf{W}^{H}[n] \mathbf{H}[n] \approx \mathbf{W}^{H}[n-1] \mathbf{H}[n]$, again the discrete Wiener-Hopf equation is the minimum, which can be computed by

$\mathbf{W}[n]=\mathbf{C}_{\boldsymbol{\alpha} \mathbf{H}}[n] \mathbf{C}_{\mathbf{H}}^{-1}[n]$

where

$$
\begin{aligned}
\mathbf{C}_{\boldsymbol{\alpha} \mathbf{H}}[n] & =\sum_{i=1}^{n} \eta^{n-i} \mathbf{H}[i] \boldsymbol{\alpha}^{H}[i] \\
& =\eta \mathbf{C}_{\mathbf{H} \boldsymbol{\alpha}}[n-1]+\mathbf{H}[n] \boldsymbol{\alpha}^{H}[n]
\end{aligned}
$$

and

$$
\begin{aligned}
\mathbf{C}_{\mathbf{H H}}[n] & =\sum_{i=1}^{n} \eta^{n-i} \mathbf{H}[i] \mathbf{H}^{H}[i] \\
& =\eta \mathbf{C}_{\mathbf{H H}}[n-1]+\mathbf{H}[n] \mathbf{H}^{H}[n] .
\end{aligned}
$$

Again, $\eta$ is a weight factor that reconsiders time variant behavior and is also set to $\eta:=0.95$.

The prediction of the CIR subspace is then straightforward RLS prediction by minimizing

$J\left(\alpha_{l}\right)=\sum_{i=1}^{n} \beta^{n-i}\left|\alpha_{l}[i+1]-\mathbf{q}_{l}^{H}[n] \boldsymbol{\alpha}_{l}[i]\right|^{2}$.

and the recursion of the filter coefficients $\mathbf{q}_{r, t}^{l}[n]$ can efficiently be calculated with

$\mathbf{q}_{l}[n]=\mathbf{q}_{l}[n-1]+\mathbf{k}_{l}[n-1] e_{l}^{*}[n]$,

where

$e_{l}[n]=\hat{\alpha}_{l}[n]-\mathbf{q}_{l}^{H}[n-1] \hat{\boldsymbol{\alpha}}_{l}[n-1]$

is the a-priori prediction error, which is given by $\hat{\alpha}_{l}[n]=\left(\alpha_{l}[n], \alpha_{l}[n-1], \ldots, \alpha_{l}\left[n-M_{P}+1\right]\right)^{T}$ that covers the history of the $l$-th CIR tap. The RLS prediction gain vector is given by

$\mathbf{k}_{l}[n]=\frac{\mathbf{P}_{l}[n-1] \hat{\boldsymbol{\alpha}}_{l}[n]}{\beta+\hat{\boldsymbol{\alpha}}_{l}^{H}[n] \mathbf{P}_{l}[n-1] \hat{\boldsymbol{\alpha}}_{l}[n]}$

and the recursively calculated, inverse of the covariance matrix of the relevant CIR taps by

$\mathbf{P}_{l}[n]=\frac{1}{\beta}\left(\mathbf{I}_{M_{P}+1}-\mathbf{k}_{l}[n] \hat{\boldsymbol{\alpha}}_{l}^{H}[n]\right) \mathbf{P}_{l}[n-1]$.

Likewise, $\beta$ is a weight factor, but it has been stated that the impact on the algorithm's convergence is very low (Schafhuber and Matz, 2005). It is set to $\beta:=0.9$.

\section{The DDCE enhancement}

In the discussed algorithm the symbols $\mathbf{s}$ are estimated by equalizing the corresponding received symbols $\tilde{\mathbf{s}}=\mathbf{H}^{-1} \mathbf{r}$. A traditional DDCE scheme would then map the symbol estimates $\tilde{\mathbf{s}}$ on the nearest constellation symbols. This can be described by a quantization operation $\mathcal{Q}: \hat{\mathbf{s}}=\mathcal{Q}\{\tilde{\mathbf{s}}\}$ which maps the infinite set of equalized symbols to the finite set of constellation symbols. The output symbol depends only on the euclidean distance of the symbol estimate to the constellation symbols as commonly used in "hard decision" demodulation. The algorithm reviewed in the previous section depends highly on correctly decided symbols $\hat{\mathbf{s}}$ that are used instead of the at the receiver unknown send symbols $\mathbf{s}$. Once a symbol is decided wrong the channel estimate is changed and further symbols are less likely to be correctly equalized. Through the PAST algorithm frequency coupling the error on a subcarrier spreads upon the others and finally the predictor outputs for all subcarriers are affected. The whole process of error propagation starts and the algorithm is unlikely to recover if not re-initialized.

Identified the weakness in the quantization it is possible to make an improvement. As said, to achieve minimum delay in processing a time varying channel, the channel coding is done on every OFDM symbol separately. Therefore, the codeword length is linked to the number of subcarriers 


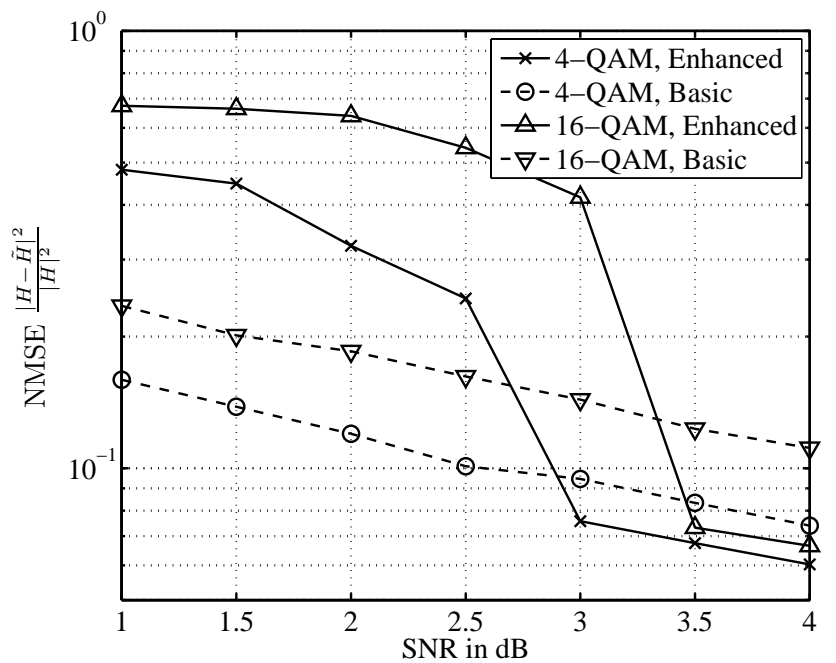

Fig. 2. Channel estimation error expressed in Normalized Mean Square Error (NMSE).

as stated in Eq. (3); the associated symbol vector of the $t$-th antenna is denoted with $\mathbf{s}^{t}$.

The single symbol mapping or quantization operation $\mathcal{Q}$ will be replaced by decisions based upon a codeword $\tilde{\mathbf{I}}=\mathcal{C}^{-1}\left\{\tilde{\mathbf{s}}^{t}\right\}$ and $\hat{\mathbf{s}}^{t}=\mathcal{C}\{\tilde{\mathbf{I}}\}$ we can write a new quantization operation as $\mathcal{Q}^{\prime}\{\cdot\}:=\mathcal{C}\left\{\mathcal{C}^{-1}\{\cdot\}\right\}$. Through the decoding and reencoding symbol errors will be corrected and the corrected versions are used to update the channel estimate in Eqs. (8) and (9).

Therefore the estimated symbols $\tilde{\mathbf{s}}^{t}$ are soft demodulated. The corresponding Log-Likelihood Ratios (LLR) $\tilde{\mathbf{I}}$ are input of the used decoder. Both Turbo Codes' and LDPC Codes' decoder use LLRs. The possibly corrected decoder output must be modulated and is then equivalent to the mapped versions $\hat{\mathbf{s}}$ and can be fed into the MIMO RLS algorithm. The whole scheme is visualized in Fig. 1.

However, if the noise is too high and too many errors occur in a codeword the underlying decoding procedure might introduce more errors through unsuccessful iterative decoding than there are originally. This is in particular the case for LDPC Codes. Thus, below the threshold of a sufficiently probable correct decoding, the proposed enhancement performs worse than the unmodified algorithm. Only above that threshold a gain in channel estimation in terms of mean square error can be realized.

\section{Simulation results}

For the following results the system parameters are given in Table 1. The MIMO transmission scheme is chosen as spatial multiplexing, i.e. two independent data streams are transmitted over the two transmit antennas. It lies in the structure of

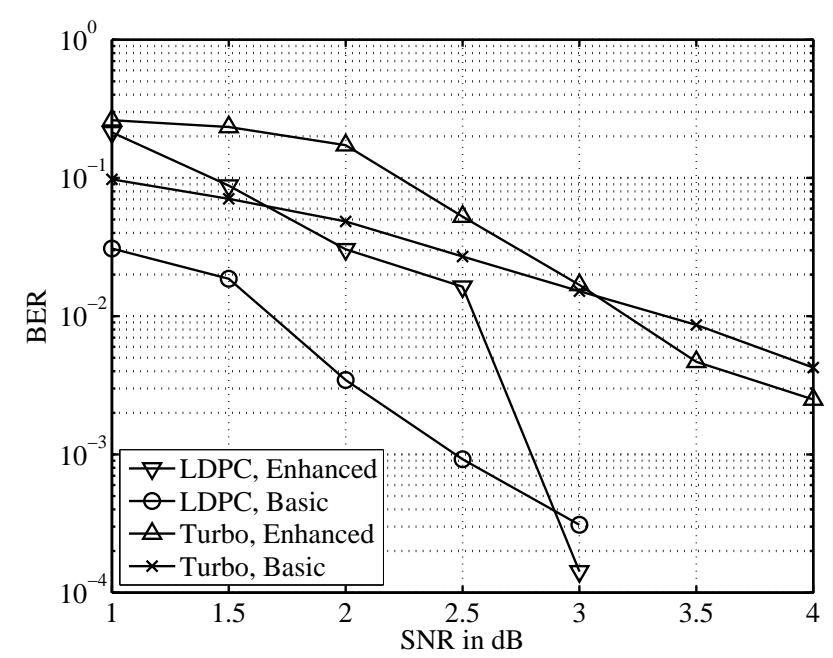

Fig. 3. BER comparison between basic DDCE and enhanced DDCE implementation for Turbo and LDPC Codes.

the MIMO RLS algorithm to find an optimal combination of the four receive signals to reconstruct the transmit signals.

\subsection{Turbo vs. LDPC codes}

In Fig. 2 a measure for the channel estimate quality, the normalized mean squared error (NMSE), is given for LDPC and Turbo Codes of $M=4$ and $M=16$ QAM. It is calculated by

$$
\mathrm{NMSE}=\frac{\sum_{n=0}^{N-1} \sum_{k=0}^{K-1} \sum_{t=1}^{n_{T}} \sum_{r=1}^{n_{R}}\left|H_{r, t}[n, k]-\tilde{H}_{r, t}[n, k]\right|^{2}}{\sum_{n=0}^{N-1} \sum_{k=0}^{K-1} \sum_{t=1}^{n_{T}} \sum_{r=1}^{n_{R}}\left|H_{r, t}[n, k]\right|} .
$$

The decoder algorithm implementation for LDPC codes is based on belief propagation (Pearl, 1988), for Turbo Codes the Log-MAP algorithm is used. Both decoder approximate a-posteriori probabilities from the soft decision inputs and decide them. For the 16-QAM modulation scheme the code rate $R_{C}$ was lowered to $1 / 4$ to match the same $E_{b} / N_{0}$ as with $4-\mathrm{QAM}$ and $R_{C}=1 / 2$. It is shown that the proposed enhancement improves the channel estimate quality in terms of MSE. All solid lines, which denote the enhanced scheme, finally are below the dashed ones, which denote the basic DDCE approach, even for 16-QAM modulation which requires higher accuracy in magnitude. The minimum of MSE is reached when also the BER in Fig. 3 first hits its minimum, i.e. error-free transmission of 15 frames. If the transmission is not error-free, in the case the SNR is too low, the proposed scheme performs much worse than the basic algorithm. This holds in terms of MSE and BER. In this case the decoding algorithms try to determine the possibly corrupted message bits from a large portion of corrupted check bits. The iterative result may end in a decoded bit word that has a larger 
Table 1. MIMO-OFDM system parameters.

\begin{tabular}{ll}
\hline$N_{T} \times N_{R}$ & $2 \times 4$ \\
number of subcarriers $K$ & 128 \\
samples of guard interval & 8 \\
number of OFDM symbols per frame & 256 \\
number of training symbols & 128 \\
channel model & COST207 BU \\
channel order $L$ & 8 \\
bandwidth & $12.5 \mathrm{MHz}$ \\
carrier frequency & $2.412 \mathrm{GHz}$ \\
normalized Doppler frequency $f_{d, n}$ & 0.0018921 \\
terminal velocity $v$ & $77 \mathrm{~km} / \mathrm{h}$
\end{tabular}

Hamming distance than the unprocessed message bits to the original message. This introduces even more symbol errors in RLS algorithm than the basic algorithm would. So applying this scheme at very low SNR yields no gain, even more loss. But above the threshold of virtually error-free decoding a gain in MSE can be realized.

\subsection{DDCE schemes vs. interpolation}

In Fig. 4 the bit error rates for three MIMO-OFDM systems (4-QAM modulated) and a single-carrier system are compared: First a MIMO-OFDM RLS enhanced DDCE system with LDPC Codes as channel coder, second again a RLS enhanced DDCE system with Turbo Codes consisting of two recursive systematic convolutional coder with generator polynomials $(7,5)$ punctured to code rate $1 / 2$. The third is a MIMO-OFDM system with a polynomial interpolation over distributed pilots in the time-frequency grid (Lieberei and Zölzer, 2008). The last system is the FS-BLAST where the interference is canceled out successively from the MIMO layers (Weikert, 2007). The number of pilots is equal to the RLS DDCE systems. Furthermore, its channel code is the same as the first system's. It is a half-rate LDPC code built from a regular parity check matrix (MacKay, 1999).

The RLS DDCE systems clearly outperform the interpolation and the FS-BLAST systems under the conditions considered here, SNR range, channel order and Doppler shifts.

\subsection{Future work}

For the simulations LDPC codes where generated using a regular parity check matrix. Recent results have shown that irregular parity check matrices have even better errorcorrecting properties (Luby et al., 1997). So additional improvements can be made if these codes are used. However, the code word length has impact on the performance. In the presented system a larger code word would need more subcarriers thus means a longer OFDM symbol duration. A larger OFDM symbol duration would decrease the ability

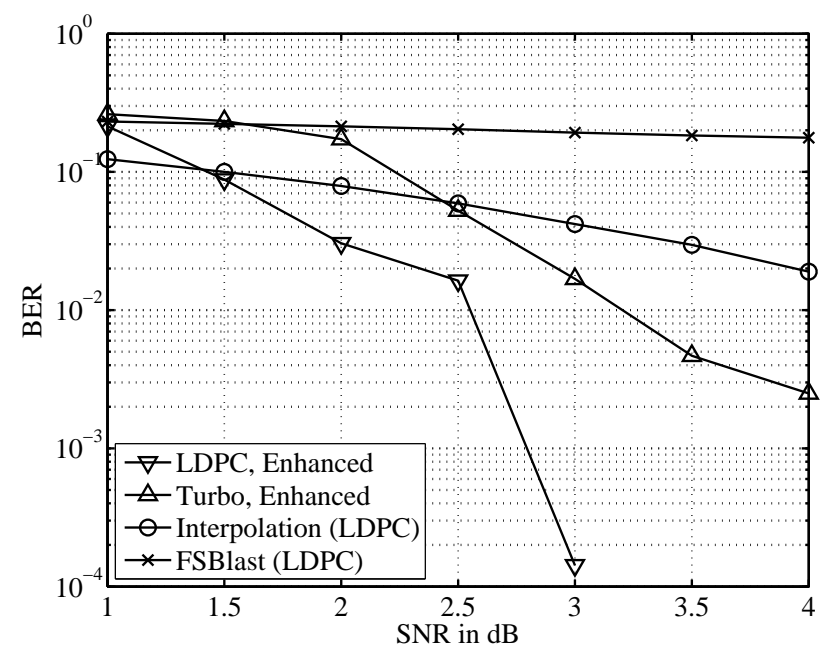

Fig. 4. BER comparison enhanced DDCE scheme with a traditional interpolation approach (LDPC coded).

to track a time-varying channel. So short and yet powerful LDPC Codes are highly desirable.

\section{Conclusions}

A channel tracking algorithm is extended for a DDCE that uses a channel decoder/encoder as a symbol quantizer. It is feasible for the algorithm to recover from errors through the error-correcting properties of the codes. This results in significant lower failure rate due to error propagation. A threshold in SNR can be determined which depends on the error-correcting performance of the applied code. Above this threshold, the proposed DDCE code enhancement improves the channel estimate and bit error rate that verifies the channel estimate. But it is limited due to the decoding operation a OFDM symbol must hold a complete code word. Below the threshold the system's performance is degraded by the proposed scheme. The choice of channel coder has impact on the overall performance.

Even LDPC Codes from regular parity check matrices outperforms widespread Turbo Codes in this context. Additionally the MIMO-RLS scheme is compared to an interpolation approach where the noise averaging ability of the RLS leads to a better bit error rate.

\section{References}

Akhtman, J. and Hanzo, L.: Advanced Channel Estimation for MIMO-OFDM in Realistic Channel Conditions, IEEE International Conference on Communications (ICC), 2528-2533, 24-28 June, 2007.

Pätzold, M.: Mobilfunkkanäle, Vieweg Verlag, 1st Edition, 1999. 
Chu, D. C.: Polyphase Codes with Good Periodic Correlation Properties, IEEE Transactions on Information Theory, 18(4), 531532, 1972.

Haykin, S.: Adaptive Filter Theory, Prentice Hall, 4th Edition, 2001.

Yang, B.: Projection Approximation Subspace Tracking, IEEE Transactions on Signal Processing, 43(1), 95-107, 1995.

Schafhuber, D. and Matz, G.: MMSE and adaptive prediction of time-varying channels for OFDM systems, IEEE Transactions on Wireless Communications, 4(2), 593-602, 2005.

Pearl, J.: Probabilistic reasoning in intelligent systems: Networks of plausible inference, Kaufmann, 1988.
Lieberei, M. and Zölzer, U.: MIMO-OFDM on a Flexible Laboratory Radio System, International Symposium on Communications, Control and Signal Processing (ISCCSP), 1184-1187, 2008.

Weikert, O.: Blinde Demodulation in MIMOÜbertragungssystemen, Dissertation, Helmut-SchmidtUniversität, 2007.

MacKay, D. J. C.: Good error-correcting codes based on very sparse matrices, IEEE Transactions on Information Theory, 45(2), 399431, 1999.

Luby, M., Mitzenmachen, M., Shokrollahi, A., Spielman, D., and Stemann, V.: Practical Loss-Resilient Codes, Proceedings of the 29th annual ACM Symposium on Theory of Computing, 150$159,1997$. 